\title{
Explanation Templates for Case-based Reasoning in Collaborative Risk Management
}

\author{
Nielsen L. R. Machado ${ }^{1}$, Lisandra M. Fontoura ${ }^{2}$, Rafael H. Bordini ${ }^{1}$, Luis A. L. Silva ${ }^{2}$ \\ ${ }^{1}$ Programa de Pós-Graduação em Ciência da Computação, PUCRS. Porto Alegre, RS, Brazil \\ nielsen.machado@acad.pucrs.br, rafael.bordini@pucrs.br \\ ${ }^{2}$ Programa de Pós-Graduação em Ciência da Computação, UFSM. Santa Maria, RS, Brazil \\ \{lisandramf, silva.luisalvaro\}@gmail.com
}

\begin{abstract}
We have put forward an approach to online collaborative discussion of software development problems based on Argumentation theory. Having records of past discussions can significantly help solve problems in new projects, and CBR techniques are used to retrieve the most similar cases. However, long discussions on past projects still contain too much information to provide support in new discussions. To address this problem, in this paper we introduce the idea of explanation templates that are able to summarize past experiences, particularly for risk management discussions. We formalize this notion of template, introduce the main templates we have developed to support explanation of past experience with risk management, and report the results of a case study on a realworld software project to assess the usefulness of those templates.
\end{abstract}

Keywords - Explanation, Argumentation, Case-Based Reasoning, Risk Management

\section{INTRODUCTION ${ }^{1}$}

Explanation and argumentation are intertwined activities in case scenarios of problem-solving. In many applications, argumentation-based dialogues [1, 2] contain rich information in the locutions exchanged by users, which in turn allow explanations to be more thorough. In this context, this work investigates a dialogue-based explanation approach [1] to select and display prominent argumentation characteristics recorded in cases for Case-Based Reasoning (CBR) systems [3]. In our project, we are particularly interested in risk management discussions conducted by stakeholders of software projects, where facts and arguments presented in discussion cases capture these risk management experiences [4-6]. As a result of queries posed by users in such CBR systems, these cases are retrieved from risk management case bases allowing such facts and arguments recorded in the most similar cases to be reused in the analysis of new problems.

To assess the content of cases in order to constructed wellgrounded experience-based solutions for new problems, different explanation techniques have been discussed in the CBR literature [7-11]. By themselves, argumentation trees recording user arguments in the structure of cases can be assessed by users as concrete narrative explanations of problem-solving situations. In a scenario in which CBR becomes a form of explanation-based reasoning, we show that explanation templates can be explored to draw users' attention to the most relevant aspects of the cases of interest, such as

${ }^{1}$ DOI reference number: 10.18293/SEKE2018-098 "highly discussed and questioned arguments", and "the balance of pro and con arguments", for instance. In our project, these argumentation characteristics are directed to the analysis of debate tasks for the identification, analysis, and response planning of risks in software projects $[12,13]$, in a scenario in which CBR supports the development of experience-based collaborative risk management tasks [4-6]. In effect, the proposed templates allow users to focus on meaningful combinations of project stakeholder moves of argumentation, as for instance, the identification of pros and cons of successful risk proposals, while other user arguments posed in these debates are temporarily omitted when cases retrieved from case bases are inspected. As a way of promoting the reuse of problem-solving information recorded in cases, these templates capture customized forms of selecting and displaying locutions defined in a dialogue protocol $[5,14]$ which is used in the collection and organization of risk management debates. As implemented in the RD System v3.0 [4], the usefulness of the templates was evaluated in two different test scenarios: a case study and a set of experiments involving different participants. The overall results show positive evidence for the usefulness of the templates proposed in the analysis and reuse of argumentation information recorded in the solution of risk management problem situations.

The paper is organized as follows. Section 2 reviews basic concepts of CBR, Argumentation, and explanation in AI. Section 3 then formalizes and informally explains what templates are and how they are used to filter the most relevant information for the users. The proposed templates along with experimental results are discussed in sections 4 and 5, respectively. Finally, final remarks are presented.

\section{BACKGROUND TO THIS WORK}

The most common form of explanation in CBR systems is related to the fact that users using these systems are able to find out the most similar cases to a current problem situation. Due to these similarity computations, as reviewed in [7], the case retrieved can offer a concrete explanation about how to make a decision on a new problem. In effect, as a past case was decided in such a way, the current problem also should be decided in this way. That is because those case situations have relevant similarities between them. In practice, past cases reflect real problem-solving situations, often providing convincing support to the conclusions that CBR systems achieve. Precedent-based explanations are also crucial in legal applications [15]. As analyzed in [8], these kinds of 
explanations are frequently attractive to users. Among other reasons, explanations grounded in past experiences are likely to be more convincing for users than explanations based on standardized explanation rules. In CBR, such explanation capabilities can be achieved when filtering and ranking methods are explored. In recommendation problems [10], for instance, besides retrieving a customized list of items, some systems also show reasons to support such recommendations.

Decision-making is subject to discussion in many CBR applications. To reach decisions, case problems can be analyzed through different user arguments. As described in [5, 14], customized dialogue protocols (or sets of locutions along with their interaction rules) can mediate risk management discussions. As organized in cases for CBR, large numbers of user arguments can be recorded in argumentation trees in the body of cases. There, the tree nodes capture the textual content of the user arguments (e.g. Fig. 1), which are indexed through the use of general kinds of locutions such as ask, inform, argument pro, argument_con, withdraw, summarize, etc. Risk management tasks also involve discussions which are particularly directed to the identification, analysis and response planning of project risks $[12,13]$. Due to this fact, a dialogue protocol to organize the development of collaborative risk management tasks can also be defined by domain-specific locutions, such as: propose risk for supporting users to pose risk statements in risk identification tasks; propose probability, propose impact to deal with risk analysis tasks; prioritize risk for the identification of the most important risks; and propose plan for the statement of risk treatment plans to deal with the prioritized risks.

\section{FormalizATION OF TEMPLATES}

Explanation templates support project stakeholders by constructing summaries of risk management discussions. Such discussions are most commonly retrieved by users when different kinds of queries are posed in CBR systems (see examples of queries in [4]). Based on standard filtering and tree-traverse techniques applied to the examination of argumentation trees, where the nodes of these trees represent user arguments advanced according to a dialogue protocol for risk management, template-based explanations can be computed. So, templates are detailed in terms of locutions used in the organization of the discussions. When templates are applied on argumentation trees represented in retrieved cases, selected parts of those discussions are separately displayed, whereas other arguments recorded are temporarily hidden. Note the importance of this combined approach for users: besides using CBR to retrieve and rank the most similar cases, users can then use templates to further examine the retrieval results so that only the most relevant arguments need to be read. We now formalize the idea of templates.

Let $\mathcal{L}$ be the set of all locutions [5] used in the argumentation-based risk management discussions stored in cases. On top of this, we describe simple expressions (very similar to regular expressions) to define a constraint over the stored arguments. We later use these constraints to formalize templates and after that we explain the semantics of each type of expression. Syntactically, the set of all constraints $C$ is inductively defined as follows:
1. if $l \in \mathcal{L}$, then $l \in C$; also _ $\in C$, where _ is a special symbol used to refer to any $l \in \mathcal{L}$.

2. if $c_{1}, c_{2} \in C$, then $\left(c_{1} \cdot c_{2}\right) \in C$; this is similar to the usual concatenation operation;

3. if $c \in C$, then $(c)$ ? $\in C$; this is the option operation, as usual (i.e., it denotes 0 or 1 occurrence of expression $c$ );

4. if $c \in C$, then $(c)^{*} \in C$; this is the repetition operation, as usual (it denotes 0 or more occurrences of expression $c$ );

5. if $l \in \mathcal{L}$, then $(l, n) \in C$, where $n \in \mathbb{N}$; this is called the threshold operation (we only display arguments within retrieved cases with at least $n$ occurrences of a locution satisfying $c$ );

6. if $l_{1}, l_{2} \in \mathcal{L}$, then $\left(l_{1}, l_{2}\right) \in C$; we call this the balance operation (the locution of interest is more frequent than its complement);

7. nothing else is in $\mathrm{C}$.

Then, formally, a template $t$ is a tuple $(i, S, D)$ where:

- $i$ is the template ID (a unique name used to refer to that template in the template base);

- $S$ is a set of selection constraints for selecting arguments within retrieved cases; a particular argument is only selected for display if it satisfies all constraints $s \in S$;

- $D$ is a set of display constraints: arguments within retrieved cases satisfying the constraints in $S$ are selected to be show to the user, but only the dialogue portions satisfying all constraints $d \in D$ are displayed.

The semantics of each kind of expression is as follows. A concatenation, such as $l_{1} \cdot l_{2}$ with $l_{1}, l_{2} \in L$, is used to refer to a dialogue excerpt where a locution $l_{2}$ is used within a dialogue context started with locution $l_{1}$. The option and repetition expressions are exactly as in regular expressions. Note that using the _operator, concatenation, and repetition we can easily define a constraint saying a particular locution may appear arbitrarily nested after another (e.g., $l_{1} \cdot * \cdot l_{2}$ ). These expressions allow for arbitrarily long but finite concatenations of any locutions, although in practice some combinations of locutions are not allowed (see [5]). However, such details are not particularly relevant to our formalization here.

The threshold operation $(l, n)$ is used to constrain the selection of arguments to those which have at least $n$ occurrences of locution $l$. In practice, $n$ is implicitly set to be the average number of occurrences of that locution in the retrieved cases. This allows, for example, expressing that only the proposal arguments with most asked questions should be selected (where by "most" we mean above average). Finally, a balance operation $\left(l_{1}, l_{2}\right) \in S$ works as follows. We require that either $l_{1} \in D$ or $l_{2} \in D$, but not both. If $l_{1} \in D$, a particular proposal argument is only selected for display if the number of occurrences of $l_{1}$ is greater than that of $l_{2}$ (and similarly if $l_{2} \in$ $D$ instead). This is useful for opposing locutions such as, for example, accepting or rejecting an argument. As an example, consider a template to select only the most questioned risk proposals, those which had the most occurrences of ask 
locutions, assuming the average number of questions typically asked is $n$. For the sake of explaining the formalization, assume we want to examine debates where the ask locution is above average but we only want the user to see the ask questions. Such a simple template could be formalized as simply $<i d_{1}$, $\{$ propose_risk $* *($ ask, $n)\},\left\{\right.$ propose_risk ${ }_{-}^{*} \cdot($ ask, $\left.n)\right\}>$.

\section{EXPLANATION TEMPLATES IN CBR SySTEMS}

Having explained how templates work and formalized them, we now proceed to present (informally only, due to space) various examples of the templates we have developed to support collaborative argumentation-based debate of ongoing risk management situations. In that context, three general types of templates can be identified, as discussed below.

The argumentation-based explanation templates select and display general purpose argumentation characteristics in argumentation trees. The template goal is detailed in terms of different arguments that appear when these dialogue protocols are used by users. To capture how and why decisions were taken, the template view of an argumentation tree is focused on properties such as highly discussed and questioned arguments and the balance of pro and con arguments. In dialogue protocols [14], for example, ask, inform, argument_pro and argument_con are standard locutions used in the identification of such argumentation characteristics.

The domain-specific explanation templates select and display domain argumentation characteristics in argumentation trees where these moves of dialogue are directly linked to the development of problem-solving tasks in certain application domains. In risk management, prominent debate tasks are the identification, analysis and response planning of risks $[12,13]$. In our dialogue protocol for risk management [5], these tasks are mainly identified when project stakeholders use propose_risk propose_impact, propose_probability and propose plan locutions, which are used in the identification of domain argumentation characteristics.

The domain and argumentation-based explanation templates select and display both domain-specific and generalpurpose argumentation characteristics in argumentation trees. In risk management, the use of such templates allows users to understand why decisions were made based on arguments. For example, debate participants can start discussions by advancing risk proposals for targeted projects. In the argumentation subtree which is rooted on such propose_risk locutions, participants can advance arguments not only to ask further information about the proposals posed originally, but also to advance pro and con arguments regarding the relevance of the risks. Having such debates retrieved from CBR queries, a template to select and display identified risks can be combined with a template to highlight proposals that were discussed by a large number of user arguments. So, the resulting template focus on propose_risk that have the largest number of ask, inform, argument_pro and argument_con locutions.

\section{A. Argumentation-based explanation templates}

Explanatory argumentation characteristics have an important role in the analysis of debates. Prominent characteristics are the balance of pros and cons for debate proposals, the fact that certain proposals may be subjected to more lengthy discussions and more heavily questioned than others, etc. Based on such properties, argumentation-based templates are proposed:

(A1) The template for the most discussed proposals selects and displays proposals because they are characterized as the most discussed in available cases. To do so, this template analyzes argumentation sub-trees rooted on propose arguments, and it checks which ones contain the highest number of user' arguments. To show the list of propose arguments to users, the decision on whether such proposals are considered as highly discussed is detailed by a threshold value in the template specification. Such decision is taken in the argumentation context of the case retrieved from a given query, since numerical criteria for deciding whether proposals are the most discussed may be different among cases.

(A2) The template for proposals with the highest number of questions asked selects and displays proposals because they are highly questioned by users. To do so, argumentation sub-trees rooted on propose arguments are identified, and the number of ask arguments in these sub-trees is considered. Then, a decision whether proposals are considered as highly questioned is determined by using a threshold value. Using an argumentation tree as input, the template displays to users only the most questioned proposals in the context of a retrieved case, where this argumentation characteristic is then displayed to users.

(A3) The template for pros and cons of proposals selects and displays proposals because their analysis is subject to argument_pro and argument_con arguments. Similarly, (A3.1) the template for proposals with the highest number of pro arguments and (A3.2) the template for proposals with the highest number of con arguments selects and displays proposals because they can be characterized as having the highest number of either pros or cons posed by users. These templates identify more arguable proposals in which users have mostly either agreed with them through the explicit use of pro arguments or disagreed with them through con arguments. To compute the outcome of such templates, argumentation subtrees rooted on propose arguments are identified, and the analysis of user arguments is focused on argument_pro and argument_con locutions only. Moreover, a threshold value is used to assess whether proposals are considered as having high numbers of pros and cons. In the end, these templates display the content of proposals along with their pros and cons.

\section{B. Domain-specific explanation templates}

Explanation templates can be related to alternative needs of explanation in targeted application domains. In risk management, these needs are directed to the identification, analysis and response planning of risks. To promote the reuse of risk information stored in cases, domain-specific explanation templates are proposed, such as the ones below.

(B1) The template for identified risk proposals selects and displays propose_risk arguments in argumentation trees because the identification of such risks is a key task for risk management activities. To do so, the content of propose_risk locutions is displayed to users. When the template is used, it hides other risk management kinds of arguments that may have 
been posed by users when dealing with risk analysis and risk response planning tasks as recorded in cases.

(B2) The template for analyzed risk proposals selects and displays propose_risk arguments along with their impact and probability analysis. To do so, it considers the propose probability and propose impact locutions within argumentation sub-trees with a propose_risk root. That is because probability and impact are commonly analyzed when determining whether a risk is prioritized in a project or not. Instead of displaying only the prioritized risks, this template selects and displays the full set of risks proposed and analyzed in debates. To target this prioritization aspect, (B2.1) the template for prioritized risk proposals selects and displays propose_risk arguments along with their impact and probability analysis provided these risks are prioritized in the project. To risk proposals in which a prioritize_risk locution is used by users, (B2.1) selects and displays such most important risks, temporally hiding other non-prioritized risks.

(B3) The template for risk response plan proposals selects and displays propose_risk arguments that have been prioritized along with their propose plan arguments, where the specification of such kinds of (mitigation, transfer, etc.) response plans are fundamental in risk management. To do so, the template displays the content of propose plan locutions for prioritized risks as recorded in cases.

(B4) The template for key risk management tasks selects and displays propose_risk arguments that have been prioritized as a result of debates. Moreover, it selects and displays propose_probability and propose_impact arguments recorded for those prioritized proposals. It also displays propose plan arguments available in the argumentation sub-trees. In effect, a debate summary is presented to users when this template is used, displaying the main arguments posed by users while identifying, analyzing and planning how to respond to risks.

\section{Domain and argumentation-based explanation templates}

To allow project stakeholders to identify risk arguments that led to more critical discussions, we created templates for selecting and displaying the most discussed, the most questioned, and the pros and cons advanced in debates regarding prioritized risks and their response plan proposals.

(C1) The template for the most discussed prioritized risk proposals selects and displays prioritized risks along with the impact and probability analysis developed by users to decide on such prioritization. It shows propose_impact and propose probability arguments, where they are recorded in argumentation sub-trees rooted on risk proposals. To measure the length of prioritized risk discussions, the number of ask, inform, argument_pro and argument_con kinds of arguments advanced by users is determined. The identification of the most discussed prioritized risks relies on those numerical estimates and defined threshold values. $(\mathrm{C} 1.1)$ The goal of the template for the most discussed risk response plan proposals is similar to the template $(\mathrm{C} 1)$, but $(\mathrm{C} 1.1)$ is focused on risks along with their treatment plan proposals. To compute the length of risk response plan debates, the template determines the number of ask, inform, argument_pro and argument_con kinds of arguments advanced by users in the debate of propose plan arguments. The determination of the most discussed plans is computed from argumentation sub-trees that are rooted on each propose plan. In the end, arguments directly related to risk and plan proposals are the only ones displayed when these templates are used.

(C2) The template for prioritized risk proposals with the highest number of questions asked selects and displays prioritized risk proposals along with question-like arguments presented by users in the analysis of these proposals. To identify the prioritized risks with the highest number of questions, the number of ask arguments advanced by users is considered. As multiple risks can be prioritized in risk management discussions, this numerical estimate is developed in the context of argumentation sub-trees directly rooted on each propose risk argument recorded in a case. (C2.1) The template for risk response plan proposals with the highest number of questions asked selects and displays prioritized risk proposals along with their risk response plan proposals. In argumentation sub-trees rooted on each propose_plan, this template counts the number of ask arguments, while this numerical estimate is considered in each one of the propose plan locutions advanced in the debates. Considering the most questioned debates, the template selects and displays the prioritized risk proposal arguments along with their risk response plan arguments. In the end, templates focusing on questions asked only display to users the content of these proposals along with questions advanced in their discussions.

(C3) The template for pro and con arguments for prioritized risk proposals selects and displays the argument_pro and argument_con arguments regarding prioritized risk proposals. When examining argumentation subtrees rooted on propose_risk, this template focuses on pros and cons arguments only. Although these pros and cons can be advanced in the debate of different risk management issues, such as in the analysis of probability and impact proposals, for example, this template only selects and displays the pros and cons that are directly related to propose_risk. Similar to (C3), (C3.1) the template for pro and con arguments for risk response plan proposals selects and displays argument_pro and argument_con arguments regarding plan proposals. These risk treatment plan arguments are captured by propose plan locutions recorded in argumentation sub-trees of prioritized risk proposals. Other templates aiming to select and display pros and cons of risk and plan proposals are: (C3.2) the template for prioritized risk proposals with the highest number of pro arguments, (C3.3) the template for risk response plan proposals with the highest number of pro arguments, (3.4) the templates for prioritized risk proposals with the highest number of con arguments and (3.5) the template for risk response plan proposals with the highest number of con arguments. In them, similar counting procedures as before are used, except now they are used to determine whether the balance between pros and cons is in favor of either pros or cons. To do so, argument_pro and argument_con arguments recorded on argumentation sub-trees rooted on propose_risk and propose_plan arguments are analyzed, respectively. As a result, only the risk and plan proposals with the highest number of pro or con arguments are displayed to users. 


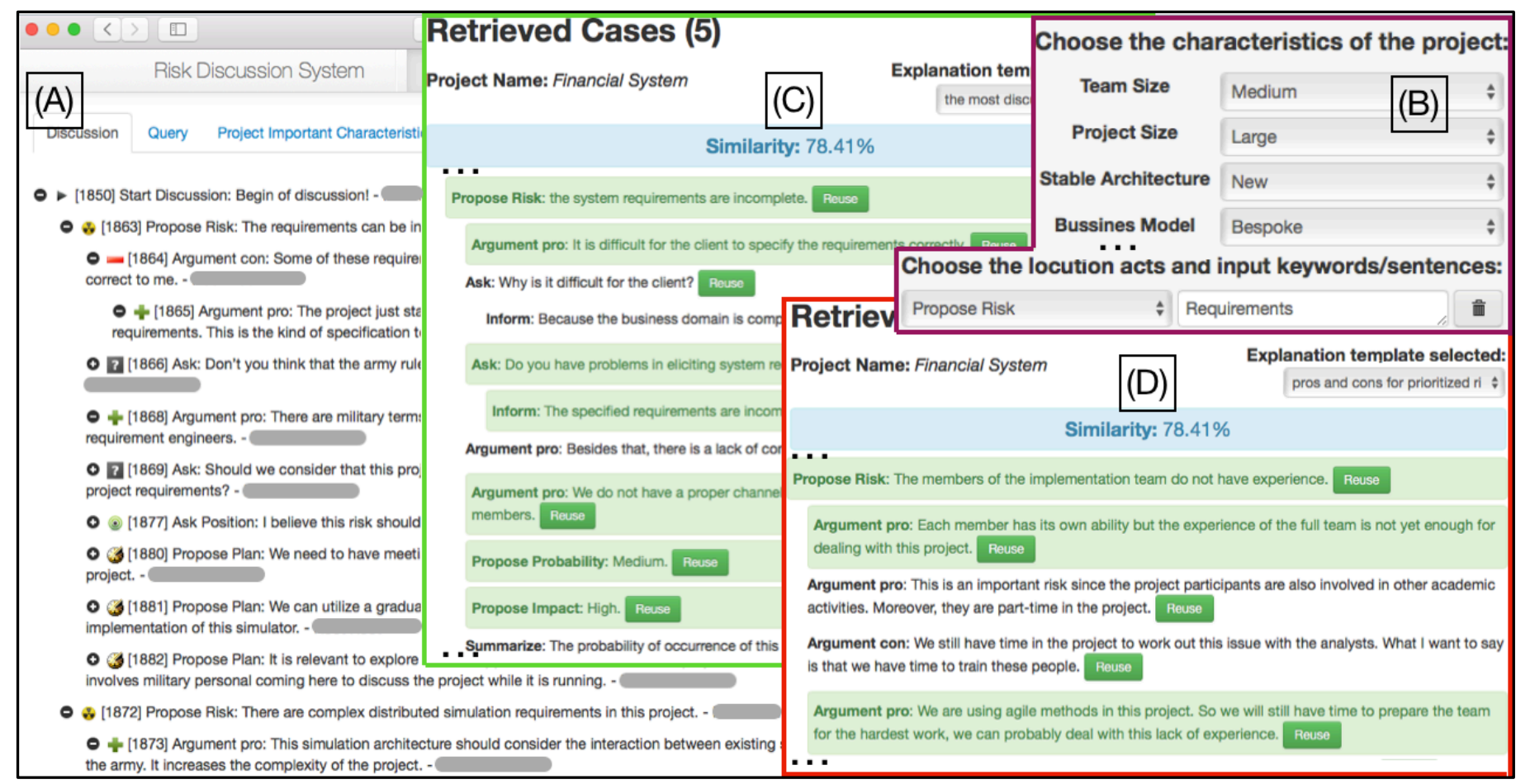

Figure 1 - A print screen of the CBR and explanation templates resources of the RD System

\section{A CASE FOR EXPLANATION TEMPLATES IN EXPERIENCE- BASED COLLABORATIVE RISK MANAGEMENT}

The usefulness of the templates proposed was assessed in a case study executed in a R\&D project aiming to build a simulation system for the Brazilian Army (e.g. [16]), where key project participants involved were a small group of researchers who were concerned with the management of risks in this project. Based on these participants' point-of-view and experience, the study goal was to examine the usefulness of the explanation-based technique detailed in this paper. Using results of queries posed by the participants in the RD System, templates were used in the analysis of the argumentation content of cases retrieved from a risk management case base.

The evaluation hypothesis $(\mathrm{H})$ and $(\mathrm{P})$ procedures were detailed in this study, where $\mathrm{H} 1$ - is the use of queries along with templates helpful in the examination of risk information in past risk management case discussions?; and P1 - to execute different queries and, for each query either use or not templates in the analysis of risk information recorded in the retrieved cases. The procedures were developed in the execution phase of this study having as a result the discussion shown in Fig. 1 (A). To H1, CBR queries based on factual information and pairs (locution, keywords) of current project were executed as in Fig. 1 (B) (see query details in [4]). Then, most of the templates were used in the filtering of the debate details recorded in the retrieved cases. First, the participants explored templates more focused on direct risk information, such as the content of prioritized risk proposals. Later, they explored templates more focused on information collected as a result of longer debates, such as the content of questions and answers (Fig. 1 (C)) and the analysis of pros and cons (Fig. 1 (D)). After that, participants analyzed the risk information that the templates were selecting and displaying in order to understand why some risks were more heavily discussed than others, for example, using the template for the most discussed prioritized risk proposals. In this scenario, some participants listed one prioritized risk from a past case retrieved, stating that such risk would be relevant in the current project. Then, they reused this risk adapting it to the current discussion (Fig. 1 (A) - argument 1863). As far as reuse of past risk information was concerned, most of the time the participants just reused the content of risk proposals. However, they also reused pro arguments to argue in the current risk management situation in favor of the occurrence of past risks examined. For that, the participants used the template for pro and con arguments for prioritized risk proposals. In this case, another risk, similar to a possible current situation was listed. Besides that, such risk contained more pro than con arguments on the past discussion. In this sense, the risk and some argument_pro were adapted on the current discussion. Finally, the participants stated that the reuse of arguments to propose and justify current risk proposals is relevant to help them to overcome debate difficulties they had, bringing more fluency to their risk management analyzes. In the evaluation phase in this case study, for these participants the use of templates allowed them to filter the kind of arguments that they wanted to examine. For them, the use of templates helped to form a quicker understanding of the debates retrieved. So, the summarization of the debates retrieved also had a positive impact on the reuse of past arguments. Finally, the participants stated that the information reviewed and examined via templates allowed them to make more informed decisions in the current problem.

To further evaluate the usefulness of the techniques proposed, $41 \mathrm{CS}$ students developed risk management tasks 
using the RD System. In these experiments, a software project was presented to the participants (divided in 9 debate groups). To assess the project risks, they formulated and executed CBR queries in the RD System. As requested, each query was repeated multiple times, allowing them to examine the content of the retrieved cases with and without the use of templates. Among a larger set of questions, they were asked to present their opinion about the following statements: (S1) The way (in particular, without using any kind of template) that past problem-solving experiences are retrieved supports (helps) the tasks of reusing such past information in the solution of the current problem. (S2) The way (in particular, now using the templates available in the system) that past problem-solving experiences are retrieved supports (helps) the tasks of reusing such past information in the solution of the current problem.

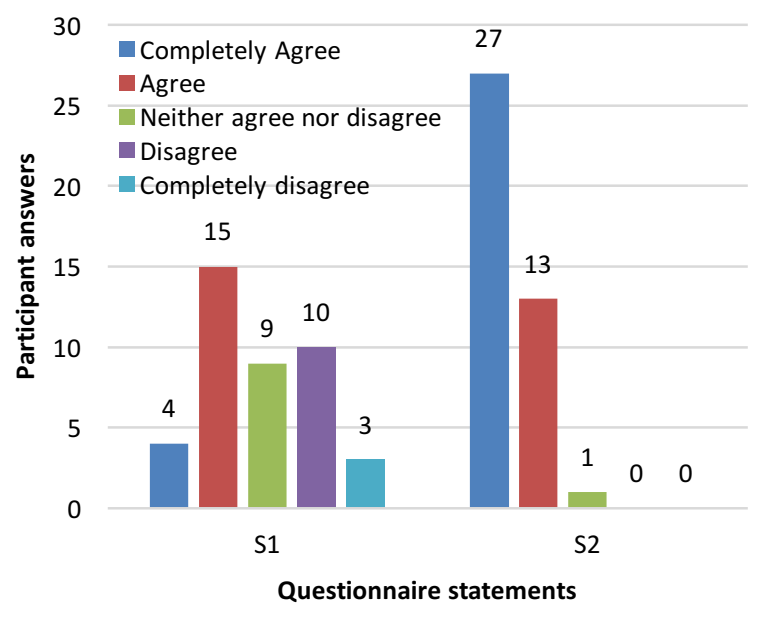

Figure 2 - Participant answers in the proposed questionnaire

According to the questionnaire results (Fig. 2), the use of templates did have a major impact on the participants' opinion with respect to the analysis and reuse of argumentation information recorded in past risk management experiences. In fact, none of the participants stated that the templates were not helpful in the reuse of past risk information recorded in the retrieved cases, even considering that they had issues when they were asked to analyze the full content of argumentation trees (see the various kinds of answers for S1).

\section{CONCLUDING REMARKS}

Remembering and explaining past risk management experiences is fundamental to the critical analysis of risks in software projects. As a way of avoiding the repetition of past risk management problems, the proposed templates allow users to examine why and how past problems were approached helping the construction of solutions for new risk management problems. In this paper we introduced a new set of explanation templates for CBR systems. We also explore the templates in the analysis and reuse of argumentation information that is recorded in cases that are not just formed by a list of facts as in standard CBR applications. Furthermore, we show the usefulness of the templates in the scenario of experience-based collaborative risk management applications. Future work involves the development of new kinds of experiments to further assess the usefulness of the explanation techniques proposed and to seek further connections with formal approaches to Argumentation in AI.

\section{ACKNOWLEDGMENT}

We gratefully acknowledge financial support from CAPESBrazil, and the Brazilian Army through the SIS-ASTROS Project (813782/2014), developed in the context of the PEE-ASTROS 2020.

\section{REFERENCES}

[1] B. Moulin et al., "Explanation and Argumentation Capabilities: Towards the Creation of More Persuasive Agents," Artificial Intelligence Review, vol. 17, no. 3, pp. 169-222, 2002.

[2] T. J. Bench-Capon, and P. E. Dunne, "Argumentation in artificial intelligence," Artificial intelligence, vol. 171 , no. 10, pp. 619-641, 2007.

[3] R. Lopez De Mantaras et al., "Retrieval, reuse, revision and retention in case-based reasoning," The Knowledge Engineering Review, vol. 20, no. 03, pp. 215-240, 2005.

[4] N. L. R. Machado et al., "Case-based Reasoning for Experiencebased Collaborative Risk Management," in The 26th Int. Conf. on Software Eng. and Knowledge Eng. (SEKE 2014), Vancouver, Canada, 2014, pp. 262-267.

[5] F. S. Severo, L. M. Fontoura, and L. A. L. Silva, "A Dialogue Game Approach to Collaborative Risk Management," in The 25th Int. Conf. on Software Eng. \& Knowledge Eng. (SEKE 2013), Boston, USA, 2013, pp. 548-551.

[6] D. L. Siqueira et al., "Argumentation Schemes for Collaborative Debate of Requirement Risks in Software Projects," Int. Journal of Software Eng. and Knowledge Eng. (IJSEKE), vol. 27, no. 910, pp. 1613-1635, 2017.

[7] F. Sørmo, J. Cassens, and A. Aamodt, "Explanation in casebased reasoning-perspectives and goals," Artificial Intelligence Review, vol. 24, no. 2, pp. 109-143, 2005.

[8] P. Cunningham, D. Doyle, and J. Loughrey, "An Evaluation of the Usefulness of Case-Based Explanation," in The 5th Int. Conf. on Case-Based Reasoning (ICCBR 2003), Trondheim, Norway, 2003, pp. 122-130.

[9] T. R. Roth-Berghofer, "Explanations and Case-Based Reasoning: Foundational Issues," in The 7th European Conf. on Case-Based Reasoning (ECCBR 2004), Madrid, Spain, 2004, pp. 389-403.

[10] D. McSherry, "Explanation in Recommender Systems," Artificial Intelligence Review, vol. 24, no. 2, pp. 179-197, 2005.

[11] R. C. Schank, Explanation Patterns: Understanding Mechanically and Creatively, Mahwah, NJ, USA: Lawrence Erlbaum Associates, Inc., 1986.

[12] A. Guide, "PROJECT MANAGEMENT BODY OF KNOWLEDGE (PMBOK® GUIDE)."

[13] B. Bohem, "Software Risk Management: Principles and Pratices," Software, IEEE, 1991.

[14] P. McBurney, and S. Parsons, "Dialogue Games for Agent Argumentation," Argumentation in Artificial Intelligence, I. Rahwan and G. R. Simari, eds., 2009.

[15] K. D. Ashley, and E. L. Rissland, "Law, Learning and Representation," Artificial Intelligence, vol. 150, no. 1-2, pp. 17$58,2003$.

[16] J. R. Brondani, E. P. Freitas, and L. A. L. Silva, "A task-oriented and parameterized (semi) autonomous navigation framework for the development of simulation systems," in The 28st Int. Conf. on Knowledge Based and Intelligent Information and Engineering Systems (KES 2017), Marseille, France, 2017, pp. 534-543. 